\title{
How We Do It: Tips for programming the speech processor of an 18-month-old
}

TERESA A ZWOLAN, BRANDI L GRIFFIN, The University of Michigan Cochlear Implant Program, Ann Arbor, Michigan, USA

\section{Introduction}

Programming the speech processor can be a challenge when working with any patient, but can be particularly challenging when working with children as young as 18 months of age. The first introduction to sound with the speech processor, often referred to as the 'activation' or 'tune-up', refers to the time when the cochlear implant is first 'turned on'. The purpose of this appointment is to introduce the child to sound with the implant and to create a baseline map from which future maps will be created. The purpose of the appointment is not to provide the child with perfect hearing. This appointment is an important starting point that has great influence on the child's success with and use of the device.

\section{Device activation}

In our clinic, the activation appointment occurs 4-6 weeks following surgery. Although this is performed sooner in some clinics, we have adhered to this time schedule as it allows an appropriate amount of time for healing of the incision and reduction of swelling around the area of the implanted receiver-stimulator. This is important, as swelling will likely interfere with adhesion of the external magnet.

Many children are resistant to wearing the speech processor on the activation day. To avoid such an issue, children are often given a 'blank' speech processor (one that contains no maps) prior to the activation appointment. When the unit is dispensed, parents are given instructions regarding proper placement of the speech processor on the child's body. It should be noted that the coil is not dispensed with the speech processor, as swelling of the incision may make adhesion of the coil difficult and placement of the coil may be uncomfortable for the child. Although this early processor provides no sound, wearing the device prior to activation helps the child become accustomed to its use, increasing the likelihood that it will be accepted and used during the activation appointment. This time also allows the parents to familiarize themselves with the equipment. 
In our clinic, the type of speech processor recommended for a patient (i.e. body processor versus ear-level processor) will depend on several factors, including the type of implant the child receives, the child's age, and the parents' ability and willingness to regularly troubleshoot the device. For some, body processors may be easier for parents and teachers to troubleshoot than an ear level processor as they have paediatric-friendly features such as alarms, visible LED screens, strong attachments to the child's body, and control knob locks. Additionally, some parents may feel that body processors are less likely to become lost or damaged than ear level processors.

When the patient arrives for the activation appointment, the incision area is assessed and the parents are queried about the child's health and wellbeing since surgery. An x-ray is obtained to evaluate placement of the electrode array. The $\mathrm{x}$ ray is reviewed by the managing audiologist prior to the initiation of programming, and is reviewed later by the surgeon. The presence of any abnormalities is noted by the audiologist, as they may affect the programming of the patient's device. This $\mathrm{x}$ ray serves as a baseline for initial device placement and may be needed for future comparison if questions arise regarding possible migration or movement of the device.

One of the most important aspects of the activation appointment is parental counselling. Although a great deal of counselling takes place prior to surgery, many parents will experience strong emotions and high anxiety levels that may cause them to forget the information they received regarding what to expect at device activation. Parents are informed that the child's first day of hearing will mimic the auditory development of a newborn child. Taking the time to discuss and review such issues will reduce many of their questions and will facilitate acceptance of the child's reaction to sound. In the long run, such discussions facilitate establishment of realistic expectations as well as hope for the future.

Parents are encouraged to be active participants in the activation of their child's device. This is accomplished by having them provide positive reinforcement to the child when he or she responds to sound. Many parents assist the audiologist with ideas and ways to help the child stay focused on a task. Thus, at least one parent is usually present in the audiologist's office throughout the activation appointment.

In our clinic, we encourage professionals and family members to attend the activation appointment if this is approved by the parents. It is important, however, not to have too many people present in the programming session as this may distract the child. Therefore, wall-mounted video cameras are present in all of our paediatric offices so observers can watch the session from another room. Additionally, the session can be videotaped and the results of the appointments can be shared with family members, schoolteachers and therapists who could not be present at the appointment.

We routinely schedule children who are less than 3 years of age to have two audiologists present for testing. One of the two audiologists consistently works 
with the family throughout the pre- and postoperative evaluation process, and is referred to as the managing audiologist. The managing audiologist oversees the programming, rehabilitation and educational needs of the child, while the assisting audiologist assists with patient management. For example, he or she will provide feedback regarding the child's responses and will assist with determination of sound field measurements.

The set-up of the clinician's office makes an important contribution to the success of the appointment. In our clinic, children are seated at child-sized tables. The patient sits directly across from the assisting audiologist with their face in view of the managing audiologist, who is seated at the programming computer. The managing audiologist positions themself to ensure that movements on the keyboard are not visible to the child. An array of age-appropriate toys are placed within hand's reach of the assisting audiologist, and placed outside the reach and view of the child.

To begin testing, the child's device is attached to the audiologist's computer via an interface unit. With all devices, testing begins with measurement of impedances across the electrode array. The responses are reviewed by the audiologist and decisions are made regarding which electrodes, if any, will be excluded from the patient's map. Open circuits or shorted electrodes, if present, are noted and are excluded from the patient's map. Impedance testing should be performed at the beginning of each appointment, as impedance measurements and status of the electrode array can change over time.

\section{Speech strategies and programming parameters}

Each manufacturer has a recommended speech-processing strategy and default parameters for use with patients. With currently available software, a simple click of the mouse prepares the software for the selected strategy. The Advanced Combination Encoder (ACE) strategy (Skinner et al., 2002) is always programmed first with Nucleus patients in our clinic. Parameters used for ACE include a stimulation rate of $900 \mathrm{~Hz}, 25 \mu$ s pulse width, maxima $=10$ and ADRO enabled. For the Med-El device, the Continuous Interleaved Sampling (CIS) strategy (Wilson et al., 1991) is used with a pulse width of $26.7 \mu$ s, extension of the frequency bands and extension of the maplaw. For the Clarion device, the High Resolution strategy (Koch et al., 2004) is used with paired stimulation, POEM features are verified and the volume dial is set to $50 / 50$ or $50 / 0$.

\section{Order of testing electrodes}

It is likely that an 18-month-old child will not participate fully in testing long enough for all of the electrodes in the array to be assessed. Therefore, clinicians usually begin testing with the expectation that only a few measurements will be possible, and that electrode information may need to be interpolated in order to 
create a map. Thus, clinicians begin by testing one of the most apical electrodes, followed by determination of threshold for a basal electrode. Testing then proceeds to every five to ten electrodes. For example, with a Nucleus device, testing may occur on electrodes 20,3 and 11, followed by assessment of electrodes 15 and 7. Testing is completed for as many electrodes as possible until the child no longer provides reliable responses. If the child is unable to respond beyond assessment of a few electrodes, interpolation can be used to create a map based on measurements obtained over different areas of the electrode array. During subsequent programming sessions, thresholds should be obtained for electrodes that were not assessed in previous sessions, and should also be reassessed for electrodes that have been tested.

\section{Test procedures}

The procedure used to obtain psychophysical measurements and create a program for the speech processor is dependent on several factors, including the child's developmental age, cognitive ability, language skills, behaviour and mood. When working with children, it is important to remain flexible and take each of these factors into consideration. As stated previously, our clinic routinely schedules two audiologists to work with children who are less than 3 years of age, increasing the likelihood that enough information will be obtained to create a program for the speech processor.

Test procedures suitable for use with an 18-month-old child include Behavioural Observation Audiometry (BOA), Visual Reinforcement Audiometry (VRA) and Conditioned Play Audiometry (CPA) (Diefendorf, 2002). BOA is used throughout the threshold- and comfort-level testing of all children and involves ongoing and continuous observation of the child's response to sound as well as the child's behaviour in the absence of sound. When a sound is presented through the cochlear implant, many children in this age group will indicate hearing by cessation of play and by presenting with a curious look on their face. Other noted behaviours include looking up, cessation of sucking, raising of eyebrows or a glance towards the parents. If these behaviours are observed in response to the stimulus, the parents and clinicians reinforce the response and encourage the child to continue listening by cheering and clapping.

In our experience, Visual Reinforcement Audiometry (VRA) testing is appropriate for use with children who are 12-32 months of age, and is also a useful tool for working with older children with cognitive delays. When using VRA, the clinician slowly increases the amount of current on an electrode until the child looks up or changes their behaviour. When this happens, the clinician will focus the child's attention to the VRA toy, which is used as a 'visual' reward for good listening. The child is distracted away from the toy, and the Houghson-Westlake technique is used to determine threshold to electrical stimulation. The procedure is repeated several times, with the current level of the signal being reduced to softer and softer levels. 
Although Conditioned Play Audiometry (CPA) is most appropriate for use with children who are 2 to 5 years of age, some 18 -month-old children will be able to participate in such testing, particularly if they have participated in multiple hearing tests. With CPA, the child is trained to complete a play activity each time a sound is heard. On the day of activation, BOA or VRA should be used first to find the level at which the child is hearing the stimulus. As the child becomes comfortable with the sound, CPA can be introduced, trained and reinforced. The types of game used to obtain a conditioned play response should be interesting and age appropriate for the child being tested. Additionally, the clinician may need to make frequent changes to the type of game being played to hold the child's attention for testing of several electrodes.

\section{Determination of threshold $(\mathrm{T})$}

The need to determine threshold information for a child will vary depending on the device used as well as on the type of speech-processing strategy selected. For example, the HiRes strategy of the Clarion device employs a software program that automatically sets thresholds to $10 \%$ of the M level (Advanced Bionics, 2004). Similar threshold levels are recommended with the Med-El CIS strategy, but must be set manually by the audiologist (Med-El CI Studio+ Software Manual). Although, threshold information is not needed to create a HiRes or Med-El CIS map, we routinely determine thresholds at device activation as such information can be used to monitor changes that may occur in the future. The Nucleus device utilizes threshold information in the ACE, CIS and SPEAK strategies (Cochlear Americas, 2005). Therefore, threshold information needs to be determined for as many electrodes as possible when working with this device.

Because young children are not familiar with the sounds provided by the implant, the behavioural responses they provide to sound on the day of activation usually occur when the stimulus is supra-threshold. Thus, threshold measurements obtained in our clinic at device activation are routinely set at levels below the level where the child first responds to sound. For Nucleus devices, thresholds are set approximately 10-15 clinical units below the level of the first behavioural response. For example, if a child first provides a reliable behavioural response at a level of 150 units, the threshold will be set to a level of 135-140 units. As the child gets older and obtains greater listening experience, the gap between the behavioural response and the level at which the threshold is set narrows. Eventually, the behavioural response will actually occur at the level where the child first hears the sound; this 'true' response level will then be used as threshold.

\section{Determination of $\mathrm{C} / \mathrm{M}$ levels}

The second type of measurement needed for creation of the patient's map is the upper level of stimulation, referred to as the comfort $(\mathrm{C})$ or most comfortable $(\mathrm{M})$ 
level. For the Nucleus devices, C level is defined as a level of stimulation that is considered to be loud but comfortable. For the Clarion and Med-El devices, M level is defined as the level where sound is most comfortable. Thus, the M level is actually softer than the $\mathrm{C}$ level. Because the overall loudness of the map is normalized for both types of measurement using live speech, the procedure used to determine the $\mathrm{C}$ or $\mathrm{M}$ level is the same for patients who are 18 months old at the time of device activation.

Children who are 18 months of age will not be able to provide the clinician with information regarding the loudness of the signal provided, unless the stimulus becomes uncomfortably loud. It is important to avoid presentation of uncomfortably loud signals, as doing so will disrupt the programming appointment and may make it impossible for testing to continue. Importantly, it is difficult to regain the child's trust if an over-stimulation occurs. Thus, it is important to proceed cautiously when evaluating supra-threshold responses.

At device activation, the $\mathrm{C}$ or $\mathrm{M}$ levels are set following completion of threshold testing. For example, when programming a Nucleus device, which utilizes $\mathrm{C}$ levels, three stimuli are presented approximately 5 units above the level where threshold was set. The level of stimulation is increased on that electrode until a dynamic range of approximately 25 units is obtained. If the child indicates that a sound is too loud (i.e. by providing a worried look or by reaching for a parent), a dynamic range of less than 25 units may be used. Simple signs of discomfort, such as crying, startling or removal of the magnet, should be noted and the $\mathrm{C} / \mathrm{M}$ level should be set below the level at which the negative response occurred. Every effort should be made to provide a dynamic range of at least 20 units. The same procedure is then used to set the $\mathrm{C} / \mathrm{M}$ levels for remaining electrodes. The electrodes are then swept at 70 and $100 \%$ of the $\mathrm{C}$ level while the child sits comfortably and plays. The child's behaviour is observed, and the $\mathrm{C}$ levels are decreased if the child appears uncomfortable. When programming an Advanced Bionics or MedEl device, which utilize M levels, three stimuli are presented above threshold, beginning with a step size of ten units. The step size is gradually decreased as the loudness is increased and approaches the expected $\mathrm{M}$ level. Testing is performed for each electrode (MedEl) or electrode band (Advanced Bionics) while the child sits quietly and interacts with the assisting audiologist. The child is watched closely to evaluate his/her reaction to sound. The level of stimulation is stopped when the child's behavior indicates that the sound is noticeably audible but not uncomfortable (i.e. strong head turn with no signs of discomfort). If the child fails to respond to stimuli on the first day, the $\mathrm{C} / \mathrm{M}$ levels are set conservatively and are modified during subsequent visits as the child adjusts to sound and becomes familiar with the programming task.

It should be kept in mind that the child's perceived loudness of programming stimuli will likely be softer than the perception that occurs when speech is introduced. Thus, it is important to remain conservative when initially setting the $\mathrm{C} / \mathrm{M}$ levels in young children. 


\section{Objective measures}

Objective measures, such as Neural Response Telemetry (NRT), Neural Response Imaging (NRI), and Electrically elicited Stapedius Reflex Thresholds (eSRTs) can be helpful when programming young children. NRT and NRI are clinically available tools that use radiofrequency telemetry technology to measure the action potentials of the auditory nerve (Shallop, Facer, and Peterson, 1999). ESRTs employ presentation of a stimulus through the implant and contralateral recording of the stapedius reflex response (Hodges, Butts, Dolan-Ash, and Balkany, 1999). Such objective measures can guide the fitting of a cochlear implant when patients are unable to perform behavioral tasks, and can provide important information about neural response and sound audibility. All three measures are routinely used in our clinic to confirm the accuracy of behaviorally-determined map settings. Such measures are also helpful when testing children for whom reliable behavioral responses can not be obtained, such as children with severe cognitive delays or other disabilities.

\section{Introduction of speech}

After completion of $\mathrm{T}$ and $\mathrm{C} / \mathrm{M}$ level testing, the child is ready for introduction of live speech. Prior to activation of the device, parents are informed that children's initial reactions to sound vary greatly and that their child may demonstrate little or no reaction, may have a negative reaction or may willingly and happily accept the sound provided by the cochlear implant. Prior to presenting live speech, the volume and sensitivity levels of the speech processor are set to the lowest possible settings. Once speech is turned on, parents are encouraged to speak to their child using a normal loudness and speaking tone. If the child becomes upset, the parents are encouraged to comfort the child while distracting them with a toy. If the child accepts the sound when the processor is at the lowest possible settings, the child's behaviour is monitored continually while the volume and sensitivity settings are gradually increased. Eventually, loud sounds are presented (clapping, laughing, etc.) to ensure the settings are appropriate. If the child appears uncomfortable, the loudness of the C/M levels are reduced until speech is comfortable, even when loud sounds are presented.

At the end of the appointment, parents receive additional counselling regarding care and use of the child's device. The parents are encouraged to provide the child with frequent, positive reinforcement when they respond to sound. They are informed that the child should wear the speech processor every waking hour, and that greater device use leads to greater success. If the child is apprehensive about wearing the device, parents are counselled to turn it down slightly but to enforce consistent use. Some parents may be hesitant to enforce device use when the child is upset. When this occurs, they should be reminded and reassured that the child is not being hurt by the implant. 


\section{Subsequent programming}

In our clinic, children are expected to return for regular programming appointments during the first year of device use, and are also expected to return annually for speech perception and speech and language evaluations. These appointments are necessary as a child's $\mathrm{T}$ and $\mathrm{C} / \mathrm{M}$ levels will change over time as the child becomes more reliable with $\mathrm{T}$ levels and adjusts to the sound of the implant. Additionally, counselling is provided during subsequent appointments regarding auditory development, auditory rehabilitation, and function and use of the speech processor.

In our clinic, the activation appointment is approximately 4-6 weeks following surgery, and includes two separate 2- or 4-hour appointments scheduled on two consecutive days. Children then return bi-weekly for the first 3 months following activation, as well as monthly during months 4, 5, 6, 9 and 12 . These appointments usually last between 1 and 2 hours. Once children have used their device for a period of 12 months, they return every 6 months for device programming or for speech perception and speech/language testing. We recommend that all patients have their device programmed at least twice a year and that they participate in speech perception and speech/language testing every 12 months. Such evaluations are essential as they provide assurance that appropriate progress is being made, which will influence recommendations for educational placement and aural rehabilitation. Importantly, such evaluations are needed to determine if the device is working properly.

During subsequent appointments, the $\mathrm{T}$ and $\mathrm{C} / \mathrm{M}$ levels are reassessed using procedures similar to those performed at device activation. Children often demonstrate greater tolerance for sounds during these appointments than at device activation. Because they cannot provide feedback regarding loudness, it is possible to set the $\mathrm{C} / \mathrm{M}$ levels too high. To avoid this, $\mathrm{C} / \mathrm{M}$ levels should be increased only if testing indicates that this is needed, as unnecessary increases in $\mathrm{C} / \mathrm{M}$ levels can have severe ramifications, including distortion of sound, adaptation of loudness and stimulation of the facial nerve.

\section{Co-ordination of programming with Short Term Auditory Therapy (STAT)}

Many of our patients travel a great distance to reach our centre. Therefore, appointments are arranged several months ahead of time. Importantly, the programming appointments are co-ordinated with appointments for the family to be seen by one of our Certified Auditory-Verbal therapists. Most children are scheduled to attend therapy on the same day they are seen by the audiologist for programming, as this facilitates their participation in a therapy programme and minimizes the time the family is away from home. These appointments have been vital to the success of many of our children as they provide the parents and the 
child with much-needed information, therapy ideas and a strong foundation for their rehabilitation.

\section{Conclusion}

At device activation, most children who are 18 months of age can provide behavioural responses to sound that facilitate creation of reliable speech processor programs. Such programs are an important starting point for successful cochlear implant use. Objective measures are available with most devices and can be used to supplement the information derived from behavioural testing and can be used to generate an early map if behavioural responses cannot be obtained. Importantly, the activation appointment provides the clinician with an opportunity to counsel parents regarding several issues, including their expectations for performance, the child's auditory and language development, care and use of the device, and the rehabilitative and educational needs of the child. Overall, the activation appointment is an important first step in the life-long process of providing sound to the child with a cochlear implant.

\section{References}

Advanced Bionics Device Fitting Manual, 2004.

Cochlear Americas, 2005 User Manual for the Nucleus Custom Sound.

Diefendorf A (2002) Detection and Assessment of Hearing Loss in Infants and Children. In Katz J (ed.) Handbook of Clinical Audiology, Fifth Edition, pp. 469-80. Baltimore, MD: Lippincott Williams \& Wilkins.

Hodges AV, Butts S, Dolan-Ash S, Balkany TJ (1999) Using electrically evoked auditory reflex thresholds to fit the CLARION cochlear implant. Annals of Otology, Rhinology and Laryngology 177 (Suppl): 64-8.

Koch DB, Osberger MJ, Segal P, Kessler D (2004) HiResolution and conventional sound processing in the HiResolution bionic ear: using appropriate outcome measures to assess speech recognition ability. Audiology and Neuro-Otology 9 (4): 214-23.

MedEl CI Studio Software Manual.

Shallop J, Facer GW, Peterson A (1999) Neural response telemetry with the Nucleus CI24M cochlear implant. Laryngoscope 109 (11): 1755-9.

Skinner M, Holden L, Whitford LA, Plant KL, Pssaros C, Holden TA (2002) Speech recognition with the Nucleus 24 SPEAK, ACE, and CIS speech coding strategies in newly implanted adults. Ear Hear 23 (3): 207-23.

Wilson B, Finley CC, Lawson DT, Wolford RD, Eddington DK, Rabinowitz WM (1991). Better speech recognition with cochlear implants. Nature 35: 236-38.

Address correspondence to: Teresa A Zwolan, 475 Market Place, Building 1, Suite A, Ann Arbor, MI 48108, USA. Tel: 734-998-8119, Fax: 734-998-8122. Email: zwolan@med.umich.edu

Accepted March 2005 